Journal Club

Editor's Note: These short reviews of recent JNeurosci articles, written exclusively by students or postdoctoral fellows, summarize the important findings of the paper and provide additional insight and commentary. If the authors of the highlighted article have written a response to the Journal Club, the response can be found by viewing the Journal Club at www.jneurosci.org. For more information on the format, review process, and purpose of Journal Club articles, please see http://jneurosci.org/content/ preparing-manuscript\#journalclub.

\title{
Does Semantic Congruency Accelerate Episodic Encoding, or Increase Semantic Elaboration?
}

\author{
Roni Tibon, Elisa Cooper, and Andrea Greve \\ Cognition and Brain Sciences Unit, MRC, Cambridge CB2 7EF, United Kingdom \\ Review of Packard et al.
}

The ability to remember new information can be influenced by the degree to which it matches previously established knowledge. This phenomenon was first described over 80 years ago by Bartlett (1932), who introduced the role of "schema" as an activated part of semantic memory that matches incoming information. It is now widely established that recognition memory is enhanced for schema-congruent information (Bower, 1972), although false memories can also increase (Brewer and Treyens, 1981).

Recent investigations have explored the neural underpinnings of schema-related effects for novel episodic memories. One framework proposes that the medial temporal lobe and the medial prefrontal cortex provide complementary routes by which new information is stored (van Kesteren et al., 2012). Nevertheless, little is known about the temporal dynamics of how schemas interact with incoming information.

A recent study in The Journal of Neuroscience by Packard et al. (2017) addresses how schemas enable better encoding of

\footnotetext{
Received Feb. 28, 2017; revised April 8, 2017; accepted April 13, 2017.

R.T. is supported by a Newton International Fellowship by the Royal Society and the British Academy. E.C. and A.G. are supported by the UK Medical Research Council (MC_A060_5PR10).

The authors declare no competing financial interests.

Correspondence should be addressed to Dr. Roni Tibon, MRC Cognition and Brain Sciences Unit, 15 Chaucer Road, Cambridge CB2 7EF, UK. E-mail: roni.tibon@mrc-cbu.cam.ac.uk.

DOI:10.1523/JNEUROSCI.0570-17.2017

Copyright $\odot 2017$ the authors $\quad 0270-6474 / 17 / 374861-03 \$ 15.00 / 0$
}

episodic information into memory, by capitalizing on the high temporal resolution of electroencephalographic (EEG) recording. Two behavioral and two EEG experiments aimed to delineate how preactivated schemas facilitate the integration of new episodic events. This is not the first EEG study of interactions between episodic and semantic memory, but previous studies have focused primarily on interactions at retrieval (Greve et al., 2007; Tibon et al., 2014) or the overlap in processing between encoding and retrieval (Bridger and Wilding, 2010; Bauch and Otten, 2012). The study by Packard et al. (2017), however, adds to the limited EEG work directed at understanding how schemas influence encoding per se.

Packard et al. (2017) used variations of the Deese-Roediger-McDermott (DRM) paradigm, which is commonly used to study false memories in humans (Deese, 1959; Roediger and McDermott, 1995). In the versions of the DRM paradigm by Packard et al. (2017), participants studied lists of four related words (e.g., "desk," "sofa," "lamp," and "chair"; except in experiment 4, where there was only one word per list), one every $250 \mathrm{~ms}$. Each list was preceded by a category cue. For some of the lists, the category cue matched the words (e.g., "furniture"; the congruent condition); for other lists, the category cue did not match the words (e.g., "colors"; the incongruent condition). During encoding, participants performed a se- mantic judgment task by indicating whether the words in the list were congruent or incongruent with the category cue (except in experiment 3; see below). Memory was assessed in a subsequent old/new recognition test for studied and unstudied single words, including new words from the same categories as the exemplars, except in experiment 4 where new words were never lures from the same category.

Two behavioral experiments (experiments 2 and 3) investigated the boundary conditions under which prior knowledge benefits memory for new events. In experiment 2, an interference task, performed either before or after semantic judgment, followed the presentation of the word lists. This did not affect the congruency advantage, suggesting that memory is facilitated before consolidation or retrieval and therefore is likely linked to on-line encoding processes. In experiment 3, participants simply pressed a button when a list of words was completed. This eliminated any congruency effects, indicating that explicit processing of semantic meaning is required for a congruency advantage to occur.

The EEG experiments (experiments 1 and 4) investigated the time course of the proposed congruency advantage. To this end, the authors contrasted event-related potentials (ERPs), recorded during encoding, for words later remembered versus those later forgotten: the "difference in memory" (Dm) effect. Two Dm effects 
were reported in experiment 1 , and replicated in experiment 4 . The first Dm effect occurred $400 \mathrm{~ms}$ after the onset of the list, revealing a significantly less negative deflection for congruent words that were subsequently remembered, relative to incongruent words that were subsequently remembered, and any subsequently forgotten words (collapsed across congruency). Hence, this early difference reflected successful memory for congruent words only. A second Dm effect occurred $\sim 600 \mathrm{~ms}$ after the stimulus: subsequently remembered words were followed by a significantly less negative deflection than forgotten words regardless of congruency, providing a more general index of memory success.

Packard et al. (2017) interpreted the early modulation as a rapid neural mechanism that acts on-line to accelerate the episodic encoding of events that are congruent with prior semantic knowledge. While this notion is consistent with some other studies (Kapur et al., 1994; Kan et al., 2009), the evidence to substantiate this claim is limited. In particular, it is not clear that the congruent condition actually improved episodic memory for individual study events. At testing, both studied items and lures showed a behavioral congruency advantage with higher hits and false alarms (Packard et al., 2017, their Fig. 1), and there was no indication that this advantage was greater for studied than for unstudied words. The authors acknowledge that congruency elevated both hits and false alarms, but they do not consider the implications of this finding for their interpretation, as follows: this could suggest a memory bias for items that are semantically related to the cue, rather than improved memory accuracy for studied events. In other words, when a test word is an exemplar of a category cue that was followed by a congruent list during study, participants may be more likely to endorse it as studied, regardless of whether they remember the word itself. This could be due to the paradigm that was used; DRM paradigms elevate false alarms and possibly encourage shared activation between semantically related items (Roediger and McDermott, 1995; Hancock et al., 2003). Hence, participants might simply have stronger category activation in the congruent condition, instead of better memory for individual words.

Do the reported ERP data contain some additional information that would allow us to disambiguate these alternative interpretations? The questions would then be what drives the pattern of neural activation at study and how is it modulated by subsequent memory effects.

During study, differences in neural activation between congruent and incongruent conditions might indicate that the activation of semantically related concepts (i.e., a schema) occurs when the first word that is presented is congruent with the category cue (e.g., "furniture: desk"). This would not occur for the first word in the incongruent condition (e.g., "color: desk"), because it does not match the category cue, although it might occur later (e.g., after the second word) in experiment 1 , as participants infer the "real" category that links the words together. In experiment 4 , where only one exemplar is presented, this would be less obvious, but still possible. The activation of semantically related concepts is likely to attenuate the N400 ERP component (Kutas and Hillyard, 1980; see also Rhodes and Donaldson, 2008), a component that reflects semantic congruency and occurs between 200 and $600 \mathrm{~ms}$ over central sites (similar to the early ERP Dm effect of Packard et al., 2017, their Figs. 2c, 3c). Because this attenuation would be greater in the congruent condition, but diminished or delayed in the incongruent condition, a difference between the two conditions would be expected. Both accounts would equally predict this congruency effect in the encoding activation, but would they make different predictions when splitting study activation by subsequent memory performance?

Indeed, Packard et al. (2017) found ERP differences between remembered and forgotten words within each condition [i.e., the ERP components were further attenuated by encoding success (the Dm effect)]. This was interpreted by the authors as an indication of accelerated event-based episodic encoding. Another possibility, however, is that the early establishment of a semantic relationship in the congruent condition enabled greater semantic elaboration on some occasions more than on others. Greater semantic elaboration is known to improve subsequent memory (the "levels of processing" effect; Craik and Lockhart, 1972) but could also increase the activation of all components within the related semantic network (i.e., both studied and unstudied items). Early attenuation of the N400 would then correlate with better subsequent memory. Such modulation does not necessitate episodic memory for the specific event. Therefore, the pattern of activation in subsequent memory does not resolve this ambiguity either.
To ensure that the Dm effects of Packard et al. (2017) are associated with episodic memory (i.e., for specific spatiotemporal events), one straightforward change to the current paradigm could be to include related lures in experiment 4. An episodic account would predict a higher ratio of hits over false alarms for congruent information. Increased false alarms for related lures are characteristic of DRM-like paradigms, especially with multiple item lists (experiment 1). If presenting a category cue followed by a single item instead of multiple ones (experiment 4) shows that false alarms interact with congruency, then this would provide compelling evidence for the engagement of episodic processes. Unfortunately, experiment 4 omitted related lures. A more decisive modification would be to introduce task demands that require memory for specific episodic events. One could use multiple lists preceded by each category cue at study. At test, pairs of words are presented, and participants are asked to discriminate whether they were studied as part of the same or different lists (a type of source memory task; Naveh-Benjamin, 2000). Accurate discrimination can be achieved only by episodic remembering of the pairing and not through stronger category activation. Then, if there was still an advantage for words from the congruent condition relative to the incongruent condition, this would suggest that a schema has enhanced memory for the specific episodic events, in this example, the occurrence of the (pairs of) words.

In summary, the finding by Packard et al. (2017) that semantic congruency accelerates differences in neural activity associated with subsequent memory is an important contribution to the literature. Interpreting this acceleration requires further investigation, particularly to what extent it specifically reflects event-based episodic encoding, and to what extent it relates to semantic memory or the concept of schema. To have additional theoretical value, the concept of a schema would seem to require more than just activating related concepts in semantic memory: a schema needs to further provide constraints that allow individual events to be remembered better. Further investigation is also needed to relate these phase-locked ERP effects to differences in oscillatory activity associated with encoding as a function of semantic congruency (Morton and Polyn, 2017) and to other frameworks predicting effects of semantic knowledge on subsequent memory (Morton and Polyn, 2016; Greve et al., 2017; 
Long and Kahana, 2017). Whatever the future findings, Packard et al. (2017) have reinforced the value of using high temporal resolution techniques to track on-line neural interactions between existing (semantic) and new (episodic) information.

\section{References}

Bartlett FC (1932) Remembering: a study in experimental and social psychology. Cambridge, UK: Cambridge UP.

Bauch EM, Otten LJ (2012) Study-Test congruency affects encoding-related brain activity for some but not all stimulus materials. J Cogn Neurosci 24:183-195. CrossRef Medline

Bower GH (1972) Mental imagery and associative learning. In: Cognition in learning and memory (Gregg L, ed), pp 51-88. New York: Wiley.

Brewer WF, Treyens JC (1981) Role of schemata in memory for places. Cogn Psychol 13:207230. CrossRef

Bridger EK, Wilding EL (2010) Requirements at retrieval modulate subsequent memory effects: an event-related potential study. Cogn Neurosci 1:254-260. CrossRef Medline

Craik FIM, Lockhart RS (1972) Levels of processing: a framework for memory research. J Verbal Learning Verbal Behav 11:671-684. CrossRef

Deese J (1959) On the prediction of occurrence of particular verbal intrusions in immediate recall. J Exp Psychol 58:17-22. CrossRef Medline
Greve A, van Rossum MC, Donaldson DI (2007) Investigating the functional interaction between semantic and episodic memory: convergent behavioral and electrophysiological evidence for the role of familiarity. Neuroimage 34:801-814. CrossRef Medline

Greve A, Cooper E, Kaula A, Anderson MC, Henson R (2017) Does prediction error drive one-shot declarative learning? J Mem Lang 94: 149-165. CrossRef

Hancock TW, Hicks JL, Marsh RL, Ritschel L (2003) Measuring the activation level of critical lures in the Deese-Roediger-McDermott paradigm. Am J Psychol 116:1-14. CrossRef Medline

Kan IP, Alexander MP, Verfaellie M (2009) Contribution of prior semantic knowledge to new episodic learning in amnesia. J Cogn Neurosci 21:938-944. CrossRef Medline

Kapur S, Craik FIM, Tulving E, Wilson AA, Houle S, Brown GM (1994) Neuroanatomical correlates of encoding in episodic memory: levels of processing effect. Proc Natl Acad Sci U S A 91:2008-2011. Medline

Kutas M, Hillyard SA (1980) Reading senseless sentences:brain potentials reflect semantic incongruity. Science 207:203-205. CrossRef Medline

Long NM, Kahana MJ (2017) Modulation of task demands suggests that semantic processing interferes with the formation of episodic associations. J Exp Psychol Learn Mem Cogn 43:167-176. CrossRef Medline

Morton NW, Polyn SM (2016) A predictive framework for evaluating models of semantic organization in free recall. J Mem Lang 86: 119-140. CrossRef Medline

Morton NW, Polyn SM (2017) Beta-band activity represents the recent past during episodic encoding. Neuroimage 147:692-702. CrossRef Medline

Naveh-Benjamin M (2000) Adult age differences in memory performance: tests of an associative deficit hypothesis. J Exp Psychol Learn Mem Cognilion 26:1170-1187. CrossRef

Packard PA, Rodríguez-Fornells A, Bunzeck N, Nicolás B, de Diego-Balaguer R, Fuentemilla L (2017) Semantic congruence accelerates the onset of the neural signals of successful memory encoding. J Neurosci 37:291-301. CrossRef Medline

Rhodes SM, Donaldson DI (2008) Association and not semantic relationships elicit the N400 effect: electrophysiological evidence from an explicit language comprehension task. Psychophysiology 45:50-59. CrossRef Medline

Roediger HL, McDermott KB (1995) Creating false memories: remembering words not presented in lists. J Exp Psychol Learn Mem Cogn 4:803-814.

Tibon R, Gronau N, Scheuplein A-L, Mecklinger A, Levy DA (2014) Associative recognition processes are modulated by the semantic unitizability of memoranda. Brain Cogn 92:1931. CrossRef

van Kesteren MT, Ruiter DJ, Fernández G, Henson RN (2012) How schema and novelty augment memory formation. Trends Neurosci 35:211-219. CrossRef Medline 